\title{
Urbanisation weakens selection on the timing of breeding and clutch size in blue tits but not in great tits
}

\author{
Claire J. Branston ${ }^{1}$ (D) Pablo Capilla-Lasheras ${ }^{1}$ (D) $\cdot$ Christopher J. Pollock $^{1,2} \cdot$ Kate Griffiths $^{1} \cdot$ Stewart White $^{1}$. \\ Davide M. Dominoni ${ }^{1}$ (1)
}

Received: 12 July 2021 / Revised: 8 October 2021 / Accepted: 12 October 2021 / Published online: 20 October 2021

(c) The Author(s) 2021

\begin{abstract}
Urbanisation is a globally occurring phenomenon and is predicted to continue increasing rapidly. Urban ecosystems present novel environments and challenges which species must acclimate or adapt to. These novel challenges alter existing or create new selection pressures on behaviours which provide an opportunity to investigate eco-evolutionary responses to contemporary environmental change. We used 7 years of breeding data from urban and forest populations of blue and great tits to understand whether selection for timing of breeding or clutch size differed between the two habitats and species. We found that urban great tits laid eggs earlier than their forest counterparts, but there was no evidence of a difference in selection for earlier breeding. Blue tits, however, did not differ in timing of egg laying between the two habitats, but selection for earlier laying was weaker in the urban environment. Both species laid smaller clutches in the urban site and had positive selection for larger clutch sizes which did not differ in strength for the great tits but did for blue tits, with weaker selection in the urban population. Our results suggest that food availability for nestlings may be constraining urban birds, and that the temporal cues females use to time breeding correctly, such as tree budburst and food availability, may be absent or reduced in urban areas due to lower caterpillar availability. These results have implications for our understanding of the adaptation of wild animals to city life.
\end{abstract}

\section{Significance statement}

Urbanisation is expanding rapidly and changing the environment many species live in. A key challenge is to understand how species adapt to the urban environment, why some species can adapt, why others cannot and what we can do to ensure that cities are ecologically sustainable and biodiversity rich. Here we show that the strength of natural selection for early breeding and larger clutch size is weaker in urban than non-urban blue tits, likely due to reduced and irregular availability of natural insect food in urban areas. This effect was not found in great tits. Thus, urbanisation can alter the selection pressures wild animals are exposed to, but this effect may differ between species, even when closely related. This has implications for our understanding of how species adapt to urban life.

Keywords Reproductive selection · Urbanisation · Adaptation $\cdot$ Life-history traits

This article is a contribution to the Topical Collection Using behavioral ecology to explore adaptive responses to anthropogenic change - Guest Editors: Jan Lindström, Constantino Macias Garcia, Caitlin Gabor

Communicated by Jan Lindström

Claire J. Branston

c.branston@hotmail.co.uk

1 Institute of Biodiversity, Animal Health and Comparative Medicine, University of Glasgow, Glasgow G12 8QQ, UK

2 School of Biology, University of Leeds, Leeds, UK

\section{Introduction}

Urbanisation is a globally increasing phenomenon that shows no signs of halting. Urban areas covered $652,825 \mathrm{~km}^{2}$ of terrestrial land globally in 2000, and it is predicted that by 2030 global urban land cover will have tripled in extent (Seto et al. 2012). In comparison to rural environments, urban areas have increased impervious surfaces, ambient temperature (heat-island effect), pollution (chemical, noise and light) and non-native species (McKinney 2002; Foley et al. 2005). The impacts of urbanisation on the phenotypes of wild organisms 
have been studied for over a century, revealing extensive phenotypic divergence between urban and rural conspecifics (Donihue and Lambert 2015; Marzluff 2016; Alberti et al. 2017). However, it is still unclear whether such phenotypic differences between urban and rural populations represent adaptive responses to urban environments and the evolutionary mechanisms that generate them (Donihue and Lambert 2015; Johnson and Munshi-South 2017; Szulkin et al. 2020; Lambert et al. 2021).

In birds, one of the most studied taxa in the field of urban evolutionary ecology, many morphological and behavioural differences have been observed between urban and rural populations. For example, urban populations often have altered foraging behaviour (Lowry et al. 2013), a wider diversity in diet (Stracey et al. 2014; Lill and Muscat 2015; Russ et al. 2015), altered vocalisation properties (Bermúdez-Cuamatzin et al. 2020; Derryberry et al. 2020) and often exhibit a smaller body size than rural counterparts (Biard et al. 2017; Caizergues et al. 2018; Strubbe et al. 2020). Similarly, differences in life-history traits between urban and rural bird populations have also been documented. Generally, urban birds lay smaller clutches, begin laying eggs earlier and have lower productivity per nesting attempt than their rural counterparts (Chamberlain et al. 2009; Sepp et al. 2018). Describing the differences in traits between urban and rural populations is an important first step in understanding species responses to urbanisation. However, there is a need to fully assess the ecological and evolutionary processes leading to phenotypic changes in urban populations (Johnson and Munshi-South 2017; Rivkin et al. 2019; Lambert et al. 2021). To this end, it is imperative to identify how variation in traits corresponds to fitness, and whether these associations differ between urban and rural populations (Ouyang et al. 2018; Szulkin et al. 2020).

Urbanisation is associated with novel environmental conditions that can lead to new selection pressures (Lowry et al. 2013; Donihue and Lambert 2015), including higher temperatures, increased light pollution and altered quality and availability of food resources (Longcore and Rich 2004; Donihue and Lambert 2015; Seress and Liker 2015). All three of these environmental factors have been linked to changes in avian life-history traits in urban populations. For example, seasonal breeders often use a combination of day length and temperature to initiate breeding (Gil and Brumm 2014). The effects of altered temperatures and the presence of light pollution in urban environments are particularly important in explaining the observed phenotypic differences between urban and rural bird populations in temperate habitats (Dominoni et al. 2020), and both factors have been linked to earlier breeding phenology in urban areas (Marciniak et al. 2007; Vaugoyeau et al. 2016; Caizergues et al. 2018; de Satgé et al. 2019; Dominoni et al. 2020). Food resources also differ between urban and rural environments, either in quantity, quality, composition and/or in their timing of availability (Pollock et al. 2017; Seress et al. 2018). Food availability is an additional cue used to initiate reproduction in many bird species; therefore, changes in the timing of resource availability in urban environments can also alter breeding phenology (Gil and Brumm 2014). Food availability also plays a central role in influencing clutch size (Price and Liou 1989). Clutch size is constrained by the amount of resources a female can find to produce and incubate eggs, the number of nestlings the parents can provide for, the survival cost to the parents in the year of breeding and the following year, and physiological constraints such as the number of eggs a female can successfully incubate (Godfray et al. 1991; Visser and Lessells 2001).

Caterpillars are a vital food resource for many seasonal breeders (Betts 1955; Burger et al. 2012; Wesołowski et al. 2019; Shutt et al. 2020), and both the quality and quantity of food delivered to nestlings are key in maximising fitness (Wilkin et al. 2009; Pollock et al. 2017). Moreover, the timing of caterpillar availability is also crucial. For example, blue tits (Cyanistes caeruleus) and great tits (Parus major) optimum breeding phenology is such that peak nestling demand coincides with a peak in caterpillar availability, which in turn is highly dependent on the timing of tree budburst (Holliday 1985; Burgess et al. 2018). Missing this optimal food window by mistiming breeding can result in negative fitness consequences (Visser and Gienapp 2019) and has led to increased selection pressures for earlier laying dates in both species (van Noordwijk et al. 1995; Visser et al. 1998; Porlier et al. 2012). However, this has mostly been studied in the context of climate change and in forest environments, while comparative evidence from urban populations is scarce (although, see Caizergues et al. 2018). Due to the increased temperature observed in urban environments (Donihue and Lambert 2015), it is possible that urbanisation could be providing similar selection pressures to climate change and could even be acting in addition to climate change.

The temporal availability of caterpillars often differs between urban and rural environments (Marciniak et al. 2007; Glądalski et al. 2017; Pollock et al. 2017; Hajdasz et al. 2019; Seress et al. 2020). For example, in urban environments caterpillar availability has been shown to be consistently lower (Marciniak et al. 2007; Glądalski et al. 2017; Pollock et al. 2017; Boyes et al. 2021), to often peak earlier in the breeding season (Hajdasz et al. 2019) and to have multiple peaks as opposed to a single strong peak observed in forest habitats (Seress et al. 2018). In two populations of blue tits in Scotland, caterpillars were more abundant in forest habitats and blue tits rearing young in forest environments fed their chicks almost exclusively on caterpillars, whereas their urban counterparts fed chicks on a variety of food items, including anthropogenic food sources, due to 
reduced caterpillar availability with reduced breeding success as a result (Pollock et al. 2017). Similar differences in caterpillar availability and blue and great tit fitness between urban and rural habitats have been found elsewhere in Europe (Glądalski et al. 2017; Seress et al. 2018, 2020). Reduced food availability or quality can also lead to lower breeding success by negatively impacting the condition of reproductive females, and in turn limiting their clutch size (Price and Liou 1989; Godfray et al. 1991) or reducing the likelihood of their offspring recruiting into the breeding population (Monrós et al. 2002). Thus, the environmental conditions found in urban habitats may modify the strength and/or direction of selection on avian life histories.

Despite decades of research on phenotypic differences between urban and rural bird populations (Chamberlain et al. 2009), relatively few studies have investigated the fitness consequences of such divergence and the extent to which divergences could arise from different selection pressures across urban and rural habitats (Senar et al. 2014; Johnson and Munshi-South 2017; Caizergues et al. 2018). Caizergues et al. (2018) present evidence for selection for earlier laying dates in forest great tits (Parus major), but not in their urban counterparts, and no selection for clutch size in the forest but positive selection in the city. They also investigated selection on morphological characteristics (tarsus, wing and tail length, as well as body mass), with only body mass in males being under negative selection, which was stronger in the urban environment. Senar et al. (2014) found that another morphological trait, the size of the black breast stripe (a sexual ornament, used in mate choice), in great tits differed between an urban and forest population and was under divergent selection.

Here we aim to investigate whether the direction and magnitude of selection in key life-history traits differs between urban and forest birds. To do so, we use a dataset spanning seven breeding seasons and comprising two sympatric species, the blue tit and great tit. Blue tits and great tits are two resident sympatric hole-nesting bird species which both evolved as forest-dwelling species (Cramp and Perrins 1993; Stenning 2018) but have readily colonised urban environments. Both species compete for resources, such as food and nesting sites, which can influence their lifehistory traits and breeding success (Dhondt 2012). These factors combined make them ideal species for investigating the effects of urbanisation on life-history traits and selection on these traits. It is worth noting, that not all studies have found differences in breeding phenology between urban and non-urban blue tits, but great tits lay consistently earlier in urban environments (Chamberlain et al. 2009). Therefore, differences in reproductive selection between the two species may be present, despite relying upon the same resources.

We first compare first egg date (FED) and clutch size of blue and great tits in one urban and one rural, forest, site. We predict that both blue and great tits residing in the urban environment will initiate egg laying earlier and have smaller clutches than their forest counterparts. Second, we investigate whether selection on first egg date and clutch size differs in direction and/or magnitude between urban and forest blue and great tits. We predict that the direction of selection on first egg date and clutch size will not differ between habitats, but selection on both first egg date and clutch size will be stronger in the forest population, due to a stronger and more defined peak in caterpillar availability in forest environments (Pollock et al. 2017). Finally, we compare food availability (caterpillar abundance and phenology) between one urban and one forest site, and test whether the degree of mismatch between blue tit peak nestling demand and the peak in caterpillar availability differs between the two habitats. This is an attempt to strengthen the argument that any difference in selection on first egg date and clutch size between urban and forest birds may be driven by food availability. We predict that caterpillars will be more readily available in the forest site compared to the urban site, and the degree of mismatch between nestling peak demand and peak food availability will be less in the forest site.

\section{Methods}

\section{Study sites, bird and caterpillar sampling}

We monitored blue and great tit populations in two sites, one forest and one urban, from 2014 until 2020. The forest site, on the east shore of Loch Lomond, Scotland, is a native oak forest $40 \mathrm{~km}$ northwest of Glasgow (Scottish Centre for Ecology and the Natural Environment, coordinates = $[56.129,-4.6145])$, and the urban site is a city centre park (Kelvingrove Park, coordinates $=[55.869,-4.2851])$, comprising a mixture of planted native and non-native tree species. In the urban site, $40 \%$ of the trees present are classified as non-native species, in comparison to $2 \%$ at the forest site (classification for native and non-native tree species taken from Patterson et al. 2014). Nest-boxes have been present at both sites in the current configuration since 2014, installed approximately $50 \mathrm{~m}$ from each other, with 161 and 66 nestboxes available at the forest and urban site respectively during our study period (2014-2020; Woodcrete, Schwegler, Germany, dimensions $=260 \mathrm{H} \times 170 \mathrm{~W} \times 180 \mathrm{D} \mathrm{cm}$, entrance hole diameter $=32 \mathrm{~mm}$ ).

Nest-boxes were monitored weekly during the nestbuilding and incubation stages. The date on which the first egg of a given clutch was laid (FED—first egg date) were directly observed in the field or back calculated if the nest was found during egg laying but before incubation commenced, assuming one egg was laid per day (Perrins 1979). FEDs were recorded as days after April; therefore, a FED 
of 1 corresponds to 1st April. Clutch size was taken as the maximum number of eggs observed once incubation had commenced, and therefore, the clutch had been completed. When clutches were expected to hatch, 14 days after incubation commenced, they were checked every other day until they hatched. The number of hatched chicks and the number of unhatched eggs were recorded on the day of hatching or the day after, depending on when the nest was checked. Attempts were made to catch breeding adults at the nest 10 days after the chicks had hatched. Although only a small number of catches were successful due to birds being wary to human disturbance in the forest population and varying catch effort among years. Thirteen days after the first egg hatched, chicks were marked with a unique metal ring (BTO, UK). After ringing nestlings, nests were not checked until at least 20 days after hatching to prevent premature fledging. At this point, any dead chicks were noted and subtracted from the total number of nestlings recorded on day 13 to give the number of chicks that fledged. It was not possible to record data blind in our study, due to our study involving focal animals observed in the field.

In 2015, caterpillars were also sampled across the two sites through branch beating five trees (two oak (Quercus spp.), two birch (Betula spp.) and one beech (Fagus spp.)) at both sites, twice weekly throughout the bird breeding season (Pollock et al. 2017). The tree species were selected for sampling to represent the dominant tree species across the two sites and due to their likelihood in hosting caterpillars (Kennedy and Southwood 1984). The effects of sampling were minimised by selecting four branches per tree, which were beaten on rotation throughout the sampling period. All arthropods were identified to order level, and Lepidoptera larvae were retained and desiccated (at $50{ }^{\circ} \mathrm{C}$ ) for $24 \mathrm{~h}$ and then weighed. The mean dry weight of caterpillars per sampling session was calculated for each site. For a full description of branch beating methods see Pollock et al. 2017.

\section{Data collation and statistical analyses}

\section{Data collation}

We filtered nesting data to only include blue and great tit breeding attempts where FED and/or clutch size were recorded from first breeding attempts. We have never recorded blue tits having second broods in our sites, and only three great tit second broods have been recorded within our sites and study period. Nesting attempts where we did not know if nestlings had fledged successfully or not were also removed. This left 602 and 150 nesting attempts for blue and great tit FED, respectively. For clutch size, our final dataset included 597 and 150 nesting attempts for blue and great tit, respectively.

\section{Statistical analyses}

All analyses were performed in R (version 3.5.1) (R Core Team 2018).

Urban and forest birds' life-history traits We first examined differences in blue and great tit's FED and clutch size between habitats using general linear models (GLMs) in the $\mathrm{R}$ package lme4 (Bates et al. 2015). Two separate GLMs were run for each species, with either FED or clutch size as the response variable. Habitat, year and their interaction were included as categorical predictors. In the two models testing for differences between habitats in clutch size, we also included FED as a continuous predictor. We also investigated whether variance in either FED and/or clutch size differed between the urban and forest populations of both species using a Fisher variance ratio test.

Additionally, we re-ran the models described above as general linear mixed models (GLMM) including female identity as a random intercept (on the subset of our blue tit data where information about female identity was available [28\% of 602 nesting observations for blue tits]). The number of females identified in our great tit dataset was too low to run a GLMM including female identity as a random intercept (only $16 \%$ of 150 nesting observations). The results found in the blue tit models with and without female identity as a random intercept did not alter the interpretation of the results (i.e. direction did not differ, and magnitude was similar in both cases). Therefore, we present results of models run on the full dataset without female identity as a random intercept here and results of models run with female identity as a random effect in the supplementary material (Table S1).

Urban and forest selection gradients on avian life-history traits The fitness measure used in this study was the number of fledglings produced in each breeding attempt. We chose to use the number of fledglings, an annual fitness measure, as opposed to lifetime reproductive success as we were unable to quantify the number of young recruited into the population, due to the difficulty we faced catching breeding adults. The number of fledglings produced in a breeding attempt could be a good proxy for lifetime reproductive success, with pairs having a high number of fledglings per breeding attempt being likely to have produced more recruits which would have a higher genetic contribution to future generations and thus increased fitness.

The number of fledglings, FED and clutch size were all standardised prior to analysis by subtracting the mean number of each in a given year from the respective variable for each habitat separately. A value of 0 is equal to the site mean in the given year, a positive value is later (FED) or greater (fledglings or clutch size) than the mean, and a negative 
value earlier (FED) or fewer (fledglings or clutch size) than the mean.

First, separate analyses for urban and forest birds were conducted to obtain linear and quadratic selection gradients for each habitat using linear mixed models. Standardised number of fledglings were used as the response variables. Standardised linear or quadratic values of either FED or clutch size, and mean FED or clutch size (dependent on the selection gradient of interest) were included as fixed effects. Nest-box number was included as a random effect.

Second, urban and forest datasets were pooled and the same model structures as in the first analyses used, but with the addition of an interaction between habitat and either standardised FED or clutch size when estimating linear selection gradients, and the addition of the interaction between habitat and the quadratic term of each trait when estimating non-linear selection gradients.

All models described above were run with and without female identity as a random intercept instead of nest-box number (due to small sample size) on a subset of the blue tit data where female identity was known. We did not do this for our great tit data due to not having enough females identified in our dataset. Due to no difference in the direction of estimates and female identity explaining little to no variation, only the linear models without female identity (the simpler models using the full dataset) being presented in the main results. Mixed models including female identity as a random intercept are presented in the supplementary material (Table S2).

\section{Mismatch between peak nestling demand and caterpillar} availability Peak caterpillar abundance was taken as the sampling date with the highest recorded caterpillar mass. Only blue tit records from 2015 were used in the following analysis, as there were not enough great tit records across the sites in this year, and caterpillar availability data was only available for 2015 .

Mismatch was calculated as the difference between the hatching date plus 10 days (peak nestling demand (Perrins 1991)) and the peak in caterpillar abundance at that site. Therefore, a positive value represents peak nestling demand was later than the caterpillar peak, zero means peak nestling demand was matched with the caterpillar peak and a negative value refers to peak nestling demand occurring earlier than the observed caterpillar peak.

To test whether the degree of mismatch between peak nestling demand and the peak in caterpillar availability differed between urban and forest blue tits, we constructed a GLM with the degree of mismatch as the response variable and habitat as a categorical fixed effect.

\section{Results}

\section{First egg date and clutch size in urban and forest populations}

Blue tits breeding in urban and forest environments showed no difference in FEDs (mean \pm SE: urban $=28.7$ $\pm 0.5, n=215$ nesting attempts, forest: $28.0 \pm 0.4, n=$ 387 nesting attempts; Table S3, Fig. 1a), but did have differences in clutch sizes (mean \pm SE: urban $=7.8 \pm 0.1$, $n=215$ nesting attempts, forest $=9.3 \pm 0.12, n=382$ nesting attempts; Table S3, Fig. 1b). On average, urban blue tits laid 1.5 fewer eggs than their forest counterparts (Table S3, Fig. 1b). There was no difference in variance in FED between urban and forest blue tits, but there was higher variance in clutch size of forest blue tits (FED: $\sigma_{\text {urban }}^{2}=46.8, \sigma_{\text {forest }}^{2}=53.3$, Fisher's $F$ test: $F_{214,386}=$ $0.87,95 \% \mathrm{CI}=0.70-1.12, p=0.29$; clutch size: $\sigma_{\text {urban }}^{2}=$ $3.36, \sigma_{\text {forest }}^{2}=5.58$, Fisher's $F$ test: $F_{214,381}=0.60,95 \% \mathrm{CI}$ $=0.48-0.77, p=<0.001)$. We found differences in breeding phenology and clutch size between great tits residing in the urban and forest sites (Table S4). Urban great tits laid their first egg 2.4 days earlier and laid 2.7 fewer eggs than their forest counterparts (Table S4, Fig. 1). The variance in FED was higher in the urban than forest great tits, but variance in clutch size did not differ between the two habitats (FED: $\sigma_{\text {urban }}^{2}=77.2, \sigma_{\text {forest }}^{2}=32.8$, Fisher's $F$ test: $F_{52,96}=2.35,95 \% \mathrm{CI}=1.48-3.88, p=<0.001$; clutch size: $\sigma_{\text {urban }}^{2}=2.41, \sigma_{\text {forest }}^{2}=3.96$, Fisher's $F$ test: $\left.F_{52,96}=0.61,95 \% \mathrm{CI}=0.38-1.00, p=0.05\right)$.

\section{Selection on first egg date and clutch size in urban and forest populations}

For both urban and forest blue tits, there was negative linear selection favouring earlier breeding (Table S5, Fig. 2a). However, the selection gradient was stronger (i.e. more negative) for forest blue tits than their urban counterparts (Table S5, Fig. 2a), with a decrease of 0.06 and 0.18 fledglings for urban and forest blue tits respectively for each day increase in FED. In contrast, great tits at the urban site were under weak negative linear selection, but not at the forest site, with no difference in the strength of selection between habitats (Table S5, Fig. 2b). There was positive linear selection favouring larger clutch sizes in both the urban and forest blue tit populations (Table S5, Fig. 3a). Selection on clutch size was significantly stronger in the forest than in the urban population (Table S5, Fig. 3a), with an increase of 0.74 fledglings for each egg laid in the forest population compared to 0.25 fledglings per egg in the urban population. However, for great tits both the 
a)

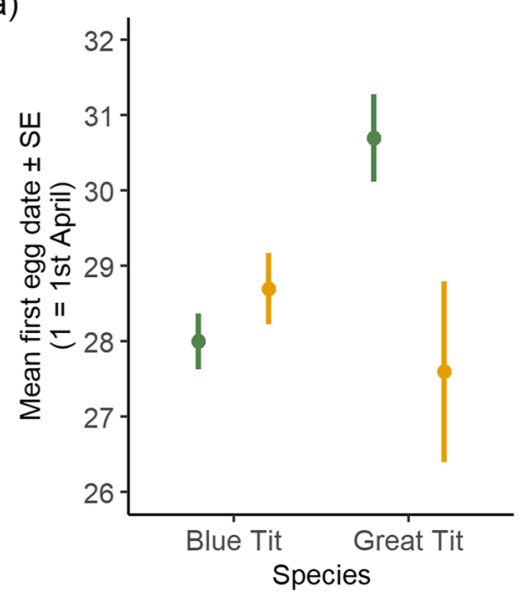

Fig. 1 First egg date and clutch size in urban and forest blue and great tits. a First egg date (in days after April 1st) of blue and great tits breeding in urban (yellow) and forest (green) habitats. Great tits in urban habitats laid eggs earlier than their forest counterparts, while urban and forest blue tits did not differ in their first egg dates. b Clutch size of blue and great tits breeding in urban and forest habi-

b)

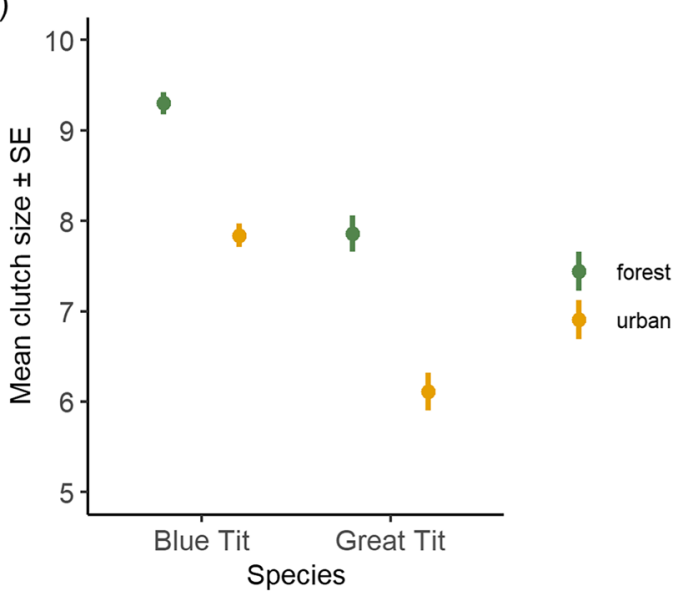

tats. In both species, individuals breeding in the urban habitat laid smaller clutches than their forest counterparts. Yellow and green points illustrate the observed mean, with the lines representing plus or minus one standard error for first egg date and clutch size in urban and forest habitats, respectively a) Blue Tit

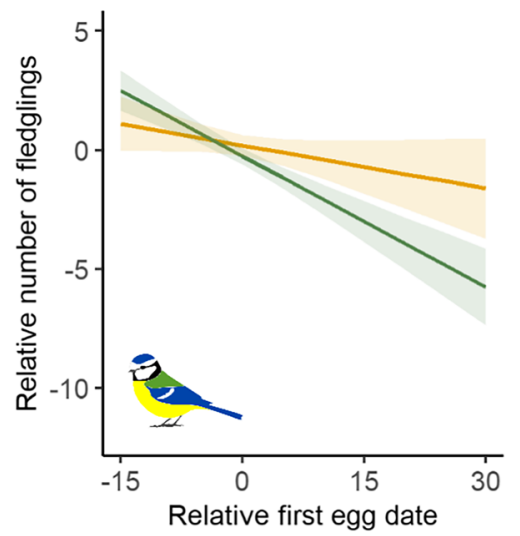

Fig. 2 Selection gradients for first egg date (FED) of urban and forest birds. FED was under negative selection in both species and in both habitats, albeit non-significant in forest great tits, where earlier clutches fledged more offspring than later clutches. a However, the strength of negative selection in urban blue tits (yellow) was significantly weaker than in the forest habitat (green; Table S5); b In great tits, the strength of selection was similar in both habitats. Solid lines represent model predictions and shaded area the 95\% confidence

urban and forest population showed positive linear selection favouring larger clutch sizes (Table S5, Fig. 3b) but there was no difference in the strength of selection between habitats (Table S5, Fig. 3b). We found no evidence for non-linear selection in either species for either FED or clutch size (Table S6). b) Great Tit

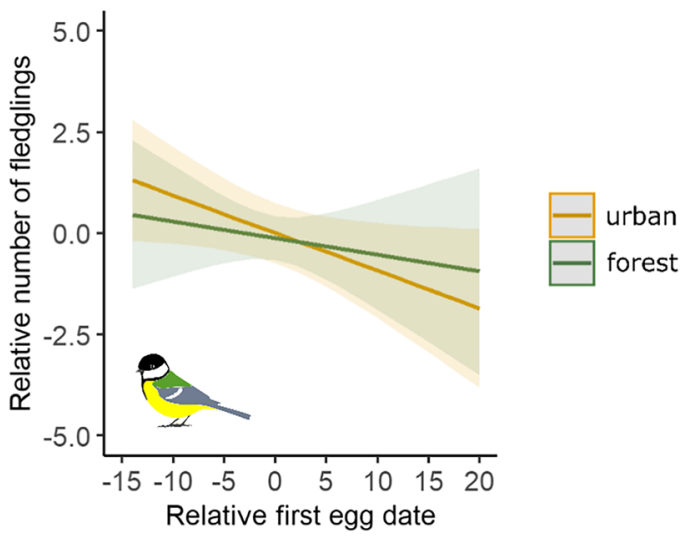

intervals from linear models with the relative number of fledglings as the response variable and relative first egg date as a predictor. All variables were standardised measures, with a value of 0 representing the mean of the variable in a given year, with a positive value representing a positive deviation (more fledglings, later first egg date) and a negative value a negative deviation (less fledglings, earlier first egg date) from the mean in the given year

\section{Caterpillar availability and mismatch with peak nestling demand}

The peak in caterpillar availability was identified as day 55 (25th May) and 72 (11th June) in the urban and forest sites respectively (Fig. 4a) during the 2015 breeding 
a) Blue Tit

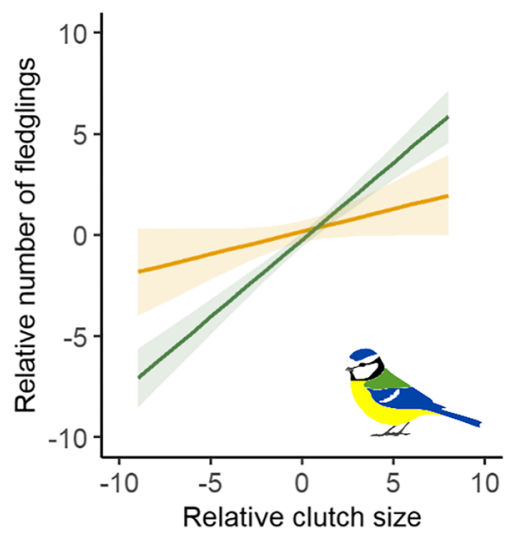

Fig. 3 Selection gradients for clutch size of urban and forest birds. Clutch size was under positive selection in both species, a blue tits and $\mathbf{b}$ great tits, and in both habitats: the larger the clutch size, the more nestlings a breeding pair were able to successfully fledge (Table S5). We only detected differences in selection gradients between habitats in blue tits (Table S5), with stronger positive selection in the forest population (green line), compared to the urban population (yellow line). Solid lines represent model predictions and b) Great Tit

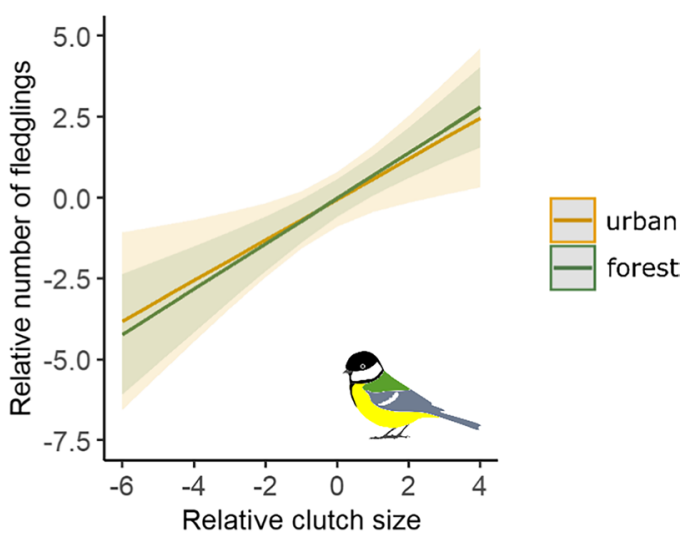

shaded area the $95 \%$ confidence intervals from linear models with the relative number of fledglings as the response variable and relative clutch size as a predictor. All variables were standardised measures, with a value of 0 representing the mean of the variable in a given year, with a positive value representing a positive deviation (more fledglings, larger clutch size) and a negative value a negative deviation (less fledglings, smaller clutch size) from the mean in the given year

a)

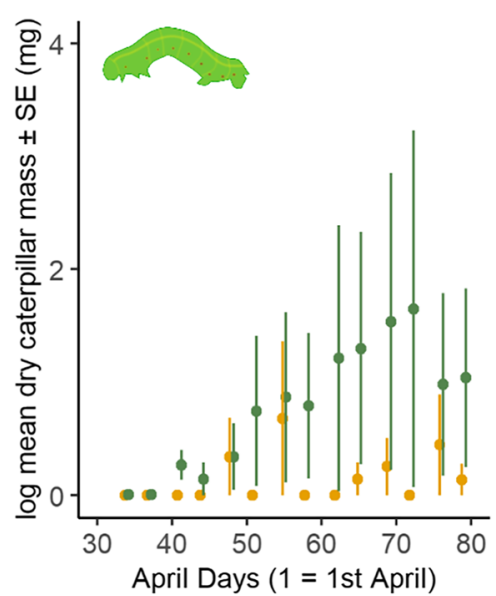

b)

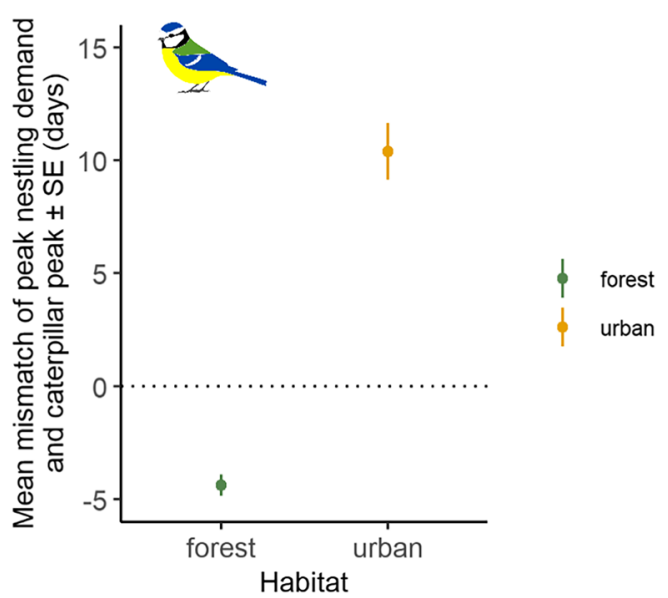

Fig. 4 Caterpillar availability and temporal mismatch between caterpillars and blue tit reproductive timing in urban and forest habitats. a Caterpillar dry weight throughout 2015 breeding season, shown here as the mean (dot) and standard error (line) of caterpillar dry weight sampled from five trees on each visit to the urban and forest site (figure adapted from Pollock et al. (2017)). b The mean (dot) and

season. Mismatch between peak food availability and nestling demand differed significantly between urban and forest sites (habitat: sum squares $=4513.3, \mathrm{df}=1,95, F=146.4$, $p=<0.001$, Fig. 4b). The peak nestling demand of forest birds was on average approximately 5 days before the peak in caterpillar availability, whereas urban birds' peak nestling demand was on average 10 days after the caterpillar peak (Fig. 4b). standard error (line) of the number of days mismatch between blue tit peak nestling demand (approximately 10 days after hatching (Perrins 1991)) and peak caterpillar availability in 2015. Peak caterpillar availability was taken as the visit with the highest mean caterpillar dry weight in $\mathbf{a}$. The dashed line in $\mathbf{b}$ represents when peak nestling demand would exactly match peak caterpillar availability

\section{Discussion}

Our results highlight that there are differences in the strength of selection between urban and forest populations, but not direction, and this differs between species and traits. We found no difference in breeding phenology (FED) between urban and forest blue tits, and variance in 
FED also did not differ. Conversely, great tits laid their first eggs earlier in the urban than forest population, and the variance in FED was also higher in the urban population. Both species laid smaller clutches in the urban population in comparison to the forest population, and there was increased variance in forest compared to urban blue tits clutch sizes. Blue tits in both the urban and forest population exhibited negative selection gradients favouring earlier egg laying, and positive selection gradients for larger clutch sizes, but selection was weaker in the urban environment for both traits. Great tits, on the other hand, exhibited weak negative selection for earlier egg laying in the urban population only, and both urban and forest populations showed positive selection for larger clutches, with no difference in the strength of selection for either trait. We also found differences between the degree of mismatch between peak nestling demand and peak caterpillar availability, with urban blue tits showing a higher degree of mismatch than their forest counterparts.

Our results showing no difference in FED between urban and forest blue tits are consistent with findings from another study at the same sites over a reduced time period (Pollock et al. 2017), a study spanning many sites across the Western Palearctic (Vaugoyeau et al. 2016) and a meta-analysis which suggested blue tits may not be consistent in their response to urban environments (Chamberlain et al. 2009). Thus, differences in blue tit FEDs between urban and forest environments may be the exception and not the norm (Chamberlain et al. 2009; Bailly et al. 2015). This may be due to habitat differences between sites described broadly as urban, but that might differ in their degree of urbanisation, amount and/or composition of tree cover, as it has been shown that lay date can vary with tree composition (Matthysen et al. 2021). Great tits, on the other hand, consistently lay earlier in urban environments in small-scale studies (Chamberlain et al. 2009; Caizergues et al. 2018), although there was no correlation between great tit FED and the intensity of urbanisation across the Western Palearctic (Bailly et al. 2015; Vaugoyeau et al. 2016).

Earlier breeding in great tits residing in urban environments compared to forest areas could be due to experiencing different cues which are used to time their breeding. Similarly to most seasonal breeders, blue and great tits use a combination of proximate (e.g. photoperiod) and supplementary cues (e.g. temperature, food availability, oak or birch leaf out) to time their breeding (Visser and Lambrechts 1999). All these cues are known to differ between the urban and forest environment. Indeed, urban environments are warmer (Kim 1992) and contain more non-native tree species than forest areas (Narango et al. 2018). Moreover, artificial lights are also widely used in cities (Kyba et al. 2017), which could potentially alter the perceived photoperiod (Dominoni et al. 2015). Such environmental differences have been generally linked to early phenology in urban animals (Neil and $\mathrm{Wu}$ 2006; Villalobos-Jiménez and Hassall 2017; Senzaki et al. 2020) and may thus explain the earlier FED found in the urban great tit population. Contrary to the results on great tits, we found no difference in blue tits' FED between our urban and forest population, and the variance in FED was not different between the two habitats. The differences we found in the two species responses to urbanisation could be explained by interspecific competition. Both species compete for the same food and nesting sites, and the larger, both in body size and mass, great tit is thought to be the more competitively dominant species out of the two (Perrins 1979). In the urban site, competition for high-quality nesting territories is likely to be fierce between the two species. Therefore, great tits may out compete blue tits and force them into lower-quality territories comprised of mixed tree species with fewer caterpillars to feed their young on, which may provide weaker cues with which to time their breeding. As such, the cues that great tits receive to time their breeding may be as strong in the urban site as the forest site, whereas blue tits may be exposed to weaker cues (Matthysen et al. 2021). Another alternative is that our results may suggest that blue tits are less sensitive than great tits to small variations in phenological cues to induce changes in phenotype in the urban population, but this hypothesis requires further investigation.

We found that selection for earlier FED in blue tits was weaker in the urban compared to the forest population. It is difficult to interpret these results within the existing literature, as to our knowledge only one study has conducted a similar analysis and only in great tits (Caizergues et al. 2018). Our findings for blue tits are similar to what was found in great tits in Caizergues et al. (2018), with stronger negative selection on FED in the forest compared to the urban population (Caizergues et al. 2018). One potential explanation for this result is that a supplementary cue used by tits to time reproductive phenology, the spring peak in caterpillar abundance, might be absent or lower in the city, leading to relaxed selection and increased variance in FED. To support this argument, in our study the availability of caterpillars was much lower and with a much less clear seasonal peak in the urban compared to the forest site. Along with reduced caterpillar availability for the nestlings in urban areas, the degree of mismatch between the small peak in urban caterpillars and peak nestling demand was greater in the urban blue tit population than the forest one, where peak nestling demand was much more synchronised with the increased caterpillar peak. The reduced height of the caterpillar peak in the urban environment could partly explain the reduced breeding success observed in urban blue tit populations (Capilla-Lasheras et al. 2017; Pollock et al. 2017; Jarrett et al. 2020; Seress et al. 2020), due to reduced availability of a highly nutritional and preferred 
food resource (Wilkin et al. 2009; Pollock et al. 2017), and could also mean that the importance of matching peak nestling demand with the caterpillar peak is weaker in the urban than in forest environments.

Surprisingly, there was no selection for early FED in forest great tits, which contradicts findings from other populations across Europe (van Noordwijk et al. 1995; Visser et al. 1998; Caizergues et al. 2018). In addition, we found no evidence for differences in selection on FED between urban and forest great tits, which differs from Caizergues et al. (2018). This highlights the difficulty in generalising results from one population to another and the need for more spatial replicates. However, it is worth noting that the selection gradients for great tits in our study are based on a much smaller sample size than those of blue tits. Indeed, breeding density of great tits at our study sites is relatively low. Scottish populations of great tits are located at the edge of their European distribution and appear to be genetically distinct from other European populations (Spurgin et al. 2019; Salmón et al. 2021), which may explain why our results differ from Caizergues et al. (2018).

Both urban blue and great tits laid smaller clutches than their forest counterparts, which is consistent with both single- and multi-site studies of these species when categorical urban versus forest comparisons were made (Bailly et al. 2015; Caizergues et al. 2018). However, no difference in clutch size of either species was found when compared with the intensity of urbanisation (Vaugoyeau et al. 2016). The reduction in clutch size we observed in these two species in urban environments could occur for three main reasons. First, the decreased caterpillar availability in urban environments discussed earlier might lead great and blue tits to lay fewer eggs, adaptatively adjusting the number of offspring to the number that they can rear given food availability at the nestling-rearing stage (Lack 1954; Price and Liou 1989). Second, decreased caterpillar availability in urban environments may constrain the female during egg laying, limiting the number of eggs she can produce due to nutrient/calcium limitation (Patten 2007). Third, interspecific competition between blue and great tits can impact upon clutch size (Dhondt 2012). Previous studies have shown a reduction in great tit clutch size when blue tit density was high (although it is worth noting that food limitations may have exacerbated this effect (Dhondt 2012)) and no effect on clutch size (Møller et al. 2018). In urban habitats, birds are often present in higher densities than in rural habitats (Møller 2009), leading to increased interspecific competition between sympatric species. In our sites, blue tit density was approximately twice as high in the forest site compared to the urban site (mean breeding pairs per $\mathrm{km}^{2}: 203$ and 107, forest and urban, respectively (CJB unpublished data)), which does not provide support for interspecific competition limiting clutch size in the urban site in either species.
Both blue and great tits in urban and forest environments showed positive selection gradients for larger clutch sizes, with the strength of selection only differing with habitat in blue tits, with weaker positive selection in the urban environment. Our great tit results are similar to Caizergues et al. (2018), who found evidence for positive selection in their urban population, as we did, but this was not the case in their forest population. The weaker selection for larger clutch sizes and reduced caterpillar availability in the urban environment we observed further suggests that resource limitation may constrain birds within urban areas to lay smaller clutches (Sepp et al. 2018; Seress et al. 2018).

We are aware of some limitations to our study and offer some scope for improvement and development in future work. First, the definition of an urban environment is difficult to compare between studies when categorical predictors are used. Therefore, it would be beneficial to compare selection on these traits along an urbanisation gradient with a quantitative assessment of urbanisation. In this context, we must acknowledge that, similarly to Caizergues et al. (2018), our study consists only of one urban and one forest population, which might differ in many other aspects other than urbanisation. Thus, comparative work on selection gradients collected across multiple urban and forest areas is needed. Second, selection gradients should be obtained from urban and forest populations of different species occupying different niches, to gain a fuller picture of the impacts of urbanisation on eco-evolutionary dynamics. Third, recent work has highlighted that selection gradients might be under-estimated unless measurement error and biological sources of within-individual variation are taken into account (Dingemanse et al. 2021). As most female individuals in our dataset are unknown, this could have underestimated our selection estimates. In a similar manner, we were also unable to account for relatedness within our analyses, which if a larger proportion of breeding adults (both male and female) were caught, could be included. Finally, we used the number of fledglings in each breeding attempt as our estimate of fitness, which could be expanded to number of recruits into the population or lifetime reproductive success. It would be interesting to compare the selection estimates calculated based on different fitness proxies to see whether these estimates are robust.

In conclusion, selection for both earlier breeding and larger clutch sizes was weaker in the urban environment in blue tits, speculatively suggesting that there may not be a strongly defined optimum time for breeding in urban environments for this species, which contrasts with the clear optimal timing of reproduction in forest environments. However, in great tits, there was no difference in the strength of selection in either trait between the two environments. This highlights the need to understand how urbanisation may be impacting different species, even sympatric urban-adapted 
species, before generalising findings from a single or few studies. Our results show that the selection pressures that species face in urban environments may be different than those in their native habitats, which has implications for our understanding of how wild animals may adapt to city life.

Supplementary Information The online version contains supplementary material available at https://doi.org/10.1007/s00265-021-03096-z.

Acknowledgements We would like to thank the many field assistants of the City Clocks team at the University of Glasgow for helping to monitor the nest-boxes and collect bird breeding data along the urbanrural gradient. We also thank the Loch Lomond and the Trossachs National Park and Glasgow City Council for allowing us access to the study areas and supporting our research. We also thank Matt Newton, Nic McCallum and Jen Page at the Scottish Centre for Ecology and the Natural Environment for facilitating our research there and two anonymous reviewers for comments which improved the manuscript.

Author contribution CJB and DMD designed the study. CJB, PC-L, CJP, KG, SW and DMD collected the data. CJB performed analyses and wrote the first draft of the manuscript. All authors commented on and approved the final manuscript before submission.

Funding This work was financially supported by a UK NERC Highlights Topics grant to DMD (NE/S005773/1) and the University of Glasgow.

Data availability The code and datasets used in this study are available from https://doi.org/10.6084/m9.figshare.14912655.

\section{Declarations}

Ethics approval This is an observational study with all research conducted following all applicable national and institutional guidelines for the use of animals and under licence from Scottish Natural Heritage (Licence Number: 156597). All bird ringing was conducted under licence from the British Trust for Ornithology, with licences to CJB, PC-L, KG, SW and DMD.

\section{Consent to participate NA.}

Consent for publication All authors approved the final manuscript before submission.

Conflict of interest The authors declare no competing interests.

Open Access This article is licensed under a Creative Commons Attribution 4.0 International License, which permits use, sharing, adaptation, distribution and reproduction in any medium or format, as long as you give appropriate credit to the original author(s) and the source, provide a link to the Creative Commons licence, and indicate if changes were made. The images or other third party material in this article are included in the article's Creative Commons licence, unless indicated otherwise in a credit line to the material. If material is not included in the article's Creative Commons licence and your intended use is not permitted by statutory regulation or exceeds the permitted use, you will need to obtain permission directly from the copyright holder. To view a copy of this licence, visit http://creativecommons.org/licenses/by/4.0/.

\section{References}

Alberti M, Marzluff J, Hunt VM (2017) Urban driven phenotypic changes: empirical observations and theoretical implications for eco-evolutionary feedback. Phil Trans R Soc 372:20160029. https://doi.org/10.1098/RSTB.2016.0029

Bailly J, Scheifler R, Berthe S, Clément-Demange V-A, Leblond M, Pasteur B, Faivre B (2015) From eggs to fledging: negative impact of urban habitat on reproduction in two tit species. J Ornithol 157:377-392. https://doi.org/10.1007/s10336-015-1293-3

Bates D, Maechler M, Bolker B, Walker S (2015) Fitting linear mixedeffects models using lme4. J Stat Softw 67:1-48. https://doi.org/ 10.18637/jss.v067.i01

Bermúdez-Cuamatzin E, Delamore Z, Verbeek L, Kremer C, Slabbekoorn H (2020) Variation in diurnal patterns of singing activity between urban and rural great tits. Front Ecol Evol 8:246. https:// doi.org/10.3389/fevo.2020.00246

Betts MM (1955) The food of titmice in oak woodland. J Anim Ecol 24:282-323. https://doi.org/10.2307/1715

Biard C, Brischoux F, Meillère A, Michaud B, Nivière M, Ruault S, Vaugoyeau M, Angelier F (2017) Growing in cities: an urban penalty for wild birds? A study of phenotypic differences between urban and rural great tit chicks (Parus major). Front Ecol Evol 5:79. https://doi.org/10.3389/fevo.2017.00079

Boyes DH, Evans DM, Fox R, Parsons MS, Pocock MJO (2021) Street lighting has detrimental impacts on local insect populations. Sci Adv 7:8322-8347. https://doi.org/10.1126/sciadv.abi8322

Burger C, Belskii E, Eeva T et al (2012) Climate change, breeding date and nestling diet: how temperature differentially affects seasonal changes in pied flycatcher diet depending on habitat variation. J Anim Ecol 81:926-936. https://doi.org/10.1111/j.1365-2656. 2012.01968.x

Burgess MD, Smith KW, Evans KL et al (2018) Tritrophic phenological match-mismatch in space and time. Nat Ecol Evol 2:970-975. https://doi.org/10.1038/s41559-018-0543-1

Caizergues AE, Grégoire A, Charmantier A (2018) Urban versus forest ecotypes are not explained by divergent reproductive selection. Proc R Soc B 285:20180261. https://doi.org/10.1098/rspb.2018. 0261

Capilla-Lasheras P, Dominoni DM, Babayan SA, O'Shaughnessy PJ, Mladenova M, Woodford L, Pollock CJ, Barr T, Baldini F, Helm B (2017) Elevated immune gene expression is associated with poor reproductive success of urban blue tits. Front Ecol Evol 5:64. https://doi.org/10.3389/fevo.2017.00064

Chamberlain DE, Cannon AR, Toms MP, Leech DI, Hatchwell BJ, Gaston KJ (2009) Avian productivity in urban landscapes: a review and meta-analysis. Ibis 151:1-18. https://doi.org/10. 1111/J.1474-919X.2008.00899.X

Cramp S, Perrins CM (1993) Handbook of the bird of Europe, the Middle East and North Africa: the birds of the Western Palearctic. Volume VII: flycatchers to shrikes. Oxford University Press, Oxford

de Satgé J, Strubbe D, Elst J, De Laet J, Adriaensen F, Matthysen E (2019) Urbanisation lowers great tit Parus major breeding success at multiple spatial scales. J Avian Biol 50:jav.02108. https://doi. org/10.1111/jav.02108

Derryberry EP, Phillips JN, Derryberry GE, Blum MJ, Luther D (2020) Singing in a silent spring: birds respond to a half-century soundscape reversion during the COVID-19 shutdown. Science 370:575-579. https://doi.org/10.1126/SCIENCE.ABD5777

Dhondt AA (2012) Interspecific competition in birds. Oxford University Press, Oxford

Dingemanse NJ, Araya-Ajoy YG, Westneat DF (2021) Most published selection gradients are underestimated: why this is and how to fix it. Evolution 75:806-818. https://doi.org/10.1111/evo.14198 
Dominoni DM, Partecke J, Partecke J (2015) Does light pollution alter daylength? A test using light loggers on free-ranging European blackbirds (Turdus merula). Phil Trans R Soc B 370:20140118. https://doi.org/10.1098/rstb.2014.0118

Dominoni DM, Kjellberg Jensen J, de Jong M, Visser ME, Spoelstra K (2020) Artificial light at night, in interaction with spring temperature, modulates timing of reproduction in a passerine bird. Ecol Appl 30:e02062. https://doi.org/10.1002/eap.2062

Donihue CM, Lambert MR (2015) Adaptive evolution in urban ecosystems. Ambio 44:194-203. https://doi.org/10.1007/ s13280-014-0547-2

Foley JA, DeFries R, Asner GP et al (2005) Global consequences of land use. Science 309:570-574. https://doi.org/10.1126/science. 1111772

Gil D, Brumm H (2014) Avian urban ecology: behavioural and physiological adaptations. Oxford University Press, Oxford

Glądalski M, Bańbura M, Kaliński A, Markowski M, Skwarska J, Wawrzyniak J, Zieliński P, Cyżewska I, Bańbura J (2017) Differences in the breeding success of blue tits Cyanistes caeruleus between a forest and an urban area: a long-term study. Acta Ornithol 52:59-68. https://doi.org/10.3161/00016454AO2017.52.1. 006

Godfray HCJ, Partridge L, Harvey PH (1991) Clutch size. Annu Rev Ecol Syst 22:409-429. https://doi.org/10.1146/annurev.es.22. 110191.002205

Hajdasz AC, Otter KA, Baldwin LK, Reudink MW (2019) Caterpillar phenology predicts differences in timing of mountain chickadee breeding in urban and rural habitats. Urban Ecosyst 22:11131122. https://doi.org/10.1007/s11252-019-00884-4

Holliday NJ (1985) Maintenance of the phenology of the winter moth (Lepidoptera: Geometridae). Biol J Linn Soc 25:221-234

Jarrett C, Powell LL, McDevitt H, Helm B, Welch AJ (2020) Bitter fruits of hard labour: diet metabarcoding and telemetry reveal that urban songbirds travel further for lower-quality food. Oecologia 193:377-388. https://doi.org/10.1007/s00442-020-04678-w

Johnson MTJ, Munshi-South J (2017) Evolution of life in urban environments. Science 358:eaam8327. https://doi.org/10.1126/scien ce.aam8327

Kennedy CEJ, Southwood TRE (1984) The number of species of insects associated with British trees: a re-analysis. J Anim Ecol 53:455-478. https://doi.org/10.2307/4528

Kim HH (1992) Urban heat island. Int J Remote Sens 13:2319-2336. https://doi.org/10.1080/01431169208904271

Kyba CCM, Kuester T, De Miguel AS, Baugh K, Jechow A, Hölker F, Bennie J, Elvidge CD, Gaston KJ, Guanter L (2017) Artificially lit surface of Earth at night increasing in radiance and extent. Sci Adv 3:e1701528. https://doi.org/10.1126/sciadv.1701528

Lack D (1954) The natural regulation of animal numbers. Oxford University Press, New York

Lambert MR, Brans KI, Des Roches S, Donihue CM, Diamond SE (2021) Adaptive evolution in cities: progress and misconceptions. Trends Ecol Evol 36:239-257. https://doi.org/10.1016/j.tree.2020. 11.002

Lill A, Muscat I (2015) Importance of inherent suitability, behavioural flexibility and competitiveness in occupancy of urban parks by an endemic honeyeater. Avian Biol Res 8:227-236. https://doi.org/ $10.3184 / 175815515 \mathrm{X} 14428542803227$

Longcore T, Rich C (2004) Ecological light pollution. Front Ecol Environ 2:191. https://doi.org/10.2307/3868314

Lowry H, Lill A, Wong BBM (2013) Behavioural responses of wildlife to urban environments. Biol Rev 88:537-549. https://doi.org/10. $1111 /$ brv. 12012

Marciniak B, Nadolski J, Nowakowska M, Loga B, Bańbura J (2007) Habitat and annual variation in arthropod abundance affects blue tit Cyanistes caeruleus reproduction. Acta Ornithol 42:53-62. https://doi.org/10.3161/068.042.0113

Marzluff JM (2016) A decadal review of urban ornithology and a prospectus for the future. Ibis 159:1-13. https://doi.org/10.1111/ibi. 12430

Matthysen E, Adriaensen F, Van De Kerckhove P, Vandekerkhove K (2021) Great and blue tit laying dates vary with fine-scale variation in local tree composition but not tree budburst. J Ornithol 162:709-722. https://doi.org/10.1007/s10336-021-01872-2

McKinney ML (2002) Urbanization, biodiversity, and conservation: the impacts of urbanization on native species are poorly studied, but educating a highly urbanized human population about these impacts can greatly improve species conservation in all ecosystems. Bioscience 52:883-890. https://doi.org/10.1641/00063568(2002)052[0883:UBAC]2.0.CO;2

Møller AP (2009) Successful city dwellers: a comparative study of the ecological characteristics of urban birds in the Western Palearctic. Oecologia 159:849-858. https://doi.org/10.1007/ s00442-008-1259-8

Møller AP, Balbontín J, Dhondt AA et al (2018) Effects of interspecific coexistence on laying date and clutch size in two closely related species of hole-nesting birds. J Anim Ecol 87:1738-1748. https:// doi.org/10.1111/1365-2656.12896

Monrós JS, Belda EJ, Barba E (2002) Post-fledging survival of individual great tits: the effect of hatching date and fledging mass. Oikos 99:481-488. https://doi.org/10.1034/j.1600-0706.2002.11909.x

Narango DL, Tallamy DW, Marra PP (2018) Nonnative plants reduce population growth of an insectivorous bird. P Natl Acad Sci USA 115:11549-11554. https://doi.org/10.1073/pnas.1809259115

Neil K, Wu J (2006) Effects of urbanization on plant flowering phenology: a review. Urban Ecosyst 9:243-257. https://doi.org/10.1007/ s11252-006-9354-2

Ouyang JQ, Isaksson C, Schmidt C, Hutton P, Bonier F, Dominoni DM (2018) A new framework for urban ecology: an integration of proximate and ultimate responses to anthropogenic change. Integr Comp Biol 58:915-928. https://doi.org/10.1093/icb/icy110

Patten MA (2007) Geographic variation in calcium and clutch size. J Avian Biol 38:637-643. https://doi.org/10.1111/j.2007.09088857.04203.x

Patterson G, Nelson D, Robertson P, Tullis J (2014) Scotland's native woodlands: results from the native woodland survey of Scotland. Forestry Commission Scotland, Edinburgh

Perrins CM (1979) British tits. Collins, London

Perrins CM (1991) Tits and their caterpillar food supply. Ibis 133:49_ 54. https://doi.org/10.1111/j.1474-919X.1991.tb07668.x

Pollock CJ, Capilla-Lasheras P, McGill RARR, Helm B, Dominoni DM (2017) Integrated behavioural and stable isotope data reveal altered diet linked to low breeding success in urban-dwelling blue tits (Cyanistes caeruleus). Sci Rep 7:5014. https://doi.org/ 10.1038/s41598-017-04575-y

Porlier M, Charmantier A, Bourgault P, Perret P, Blondel J, Garant D (2012) Variation in phenotypic plasticity and selection patterns in blue tit breeding time: between- and within-population comparisons. J Anim Ecol 81:1041-1051. https://doi.org/10.1111/j. 1365-2656.2012.01996.x

Price T, Liou L (1989) Selection on clutch size in birds. Am Nat 134:950-959. https://doi.org/10.1086/285023

Core Team R (2018) R: a language and environment for statistical computing. R Foundation for Statistical Computing, Vienna http:// www.R-project.org

Rivkin LR, Santangelo JS, Alberti M et al (2019) A roadmap for urban evolutionary ecology. Evol Appl 12:384-398. https://doi.org/10. 1111/eva.12734

Russ A, Rüger A, Klenke R (2015) Seize the night: European blackbirds (Turdus merula) extend their foraging activity under 
artificial illumination. J Ornithol 156:123-131. https://doi.org/ 10.1007/s10336-014-1105-1

Salmón P, Jacobs A, Ahrén D et al (2021) Continent-wide genomic signatures of adaptation to urbanisation in a songbird across Europe. Nat Commun 12:2983. https://doi.org/10.1038/ s41467-021-23027-w

Senar JC, Conroy MJ, Quesada J, Mateos-Gonzalez F (2014) Selection based on the size of the black tie of the great tit may be reversed in urban habitats. Ecol Evol 4:2625-2632. https://doi.org/10.1002/ ece3.999

Senzaki M, Barber JR, Phillips JN et al (2020) Sensory pollutants alter bird phenology and fitness across a continent. Nature 587:605609. https://doi.org/10.1038/s41586-020-2903-7

Sepp T, McGraw KJ, Kaasik A, Giraudeau M (2018) A review of urban impacts on avian life-history evolution: does city living lead to slower pace of life? Glob Change Biol 24:1452-1469. https://doi. org/10.1111/gcb.13969

Seress G, Liker A (2015) Habitat urbanization and its effects on birds. Acta Zool Hung 61:373-408. https://doi.org/10.17109/AZH.61.4. 373.2015

Seress G, Hammer T, Bókony V, Vincze E, Preiszner B, Pipoly I, Sinkovics C, Evans KL, Liker A (2018) Impact of urbanization on abundance and phenology of caterpillars and consequences for breeding in an insectivorous bird. Ecol Appl 28:1143-1156. https://doi.org/10.1002/eap.1730

Seress G, Sándor K, Evans KL, Liker A (2020) Food availability limits avian reproduction in the city: an experimental study on great tits Parus major. J Anim Ecol 89:1570-1580. https://doi.org/10.1111/ 1365-2656.13211

Seto KC, Güneralp B, Hutyra LR (2012) Global forecasts of urban expansion to 2030 and direct impacts on biodiversity and carbon pools. P Natl Acad Sci USA 109:16083-16088. https://doi.org/ 10.1073/pnas.1211658109

Shutt JD, Nicholls JA, Trivedi UH, Burgess MD, Stone GN, Hadfield JD, Phillimore AB (2020) Gradients in richness and turnover of a forest passerine's diet prior to breeding: a mixed model approach applied to faecal metabarcoding data. Mol Ecol 29:1199-1213. https://doi.org/10.1111/mec.15394

Spurgin LG, Bosse M, Adriaensen F et al (2019) The great tit HapMap project: a continental-scale analysis of genomic variation in a songbird. bioRxiv 14:21. https://doi.org/10.1101/561399

Stenning M (2018) The blue tit. T \& AD Poyser, London

Stracey CM, Wynn B, Robinson SK (2014) Light pollution allows the northern mockingbird (Mimus polyglottos) to feed nestlings after dark. Wilson J Ornithol 126:366-369. https://doi.org/10.1676/ 13-107.1
Strubbe D, Salleh Hudin N, Teyssier A, Vantieghem P, Aerts J, Lens L (2020) Phenotypic signatures of urbanization are scale-dependent: a multi-trait study on a classic urban exploiter. Landsc Urban Plan 197:103767. https://doi.org/10.1016/j.landurbplan.2020.103767

Szulkin M, Munshi-South J, Charmantier A (2020) Urban evolutionary biology. Oxford University Press, Oxford

van Noordwijk AJ, McCleery RH, Perrins CM, van Noordwijk AJ, McCleery RH, Perrins CM (1995) Selection for the timing of great tit breeding in relation to caterpillar growth and temperature. J Anim Ecol 64:451-458. https://doi.org/10.2307/5648

Vaugoyeau M, Adriaensen F, Artemyev A et al (2016) Interspecific variation in the relationship between clutch size, laying date and intensity of urbanization in four species of hole-nesting birds. Ecol Evol 6:5907-5920. https://doi.org/10.1002/ece3.2335

Villalobos-Jiménez G, Hassall C (2017) Effects of the urban heat island on the phenology of Odonata in London, UK. Int J Biometeorol 61:1337-1346. https://doi.org/10.1007/s00484-017-1311-7

Visser ME, Gienapp P (2019) Evolutionary and demographic consequences of phenological mismatches. Nat Ecol Evol 3:879-885. https://doi.org/10.1038/s41559-019-0880-8

Visser ME, Lambrechts MM (1999) Information constraints in the timing of reproduction in temperate zone birds: great and blue tits. In: Adams NJ, Slotow RH (eds) Proceedings of the 22nd international ornithological congress, Durban. BirdLife South Africa, Johannesburg, pp 249-264

Visser ME, Lessells CM (2001) The costs of egg production and incubation in great tits (Parus major). Proc R Soc Lond B 268:12711277. https://doi.org/10.1098/rspb.2001.1661

Visser ME, van Noordwijk AJ, Tinbergen JM, Lessells CM (1998) Warmer springs lead to mistimed reproduction in great tits (Parus major). Proc R Soc Lond B 265:1867-1870. https://doi.org/10. 1098/rspb.1998.0514

Wesołowski T, Rowiński P, Neubauer G (2019) Food of nuthatch Sitta europaea young in a primeval forest: effects of varying food supply and age of nestlings. Acta Ornithol 54:85-104. https://doi.org/ 10.3161/00016454AO2019.54.1.008

Wilkin TA, King LE, Sheldon BC (2009) Habitat quality, nestling diet, and provisioning behaviour in great tits Parus major. J Avian Biol 40:135-145

Publisher's note Springer Nature remains neutral with regard to jurisdictional claims in published maps and institutional affiliations. 